\title{
Human Rabies — Puerto Rico, 2015
}

Ashley Styczynski, MD ${ }^{1,2}$; Cuc Tran, PhD ${ }^{1,2}$; Emilio Dirlikov, PhD ${ }^{1,3}$; María Ramos Zapata, MPH³; Kyle Ryff, MPH³; Brett Petersen, MD²; Anibal Cruz Sanchez, $\mathrm{MPH}^{3}$; Marrielle Mayshack ${ }^{3,4}$; Laura Castro Martinez, $\mathrm{MPH}^{3}$; Rene Condori, MS ${ }^{2}$; James Ellison, $\mathrm{PhD}^{2}$; Lillian Orciari, MS²; Pamela Yager ${ }^{2}$; Rafael González Peña ${ }^{3}$; Dario Sanabria, MD5; Julio Cádiz Velázquez, MD³; Dana Thomas, MD³, Brenda Rivera García, DVM³

On December 1, 2015, the Puerto Rico Department of Health (PRDH) was notified by a local hospital of a suspected human rabies case. The previous evening, a Puerto Rican man aged 54 years arrived at the emergency department with fever, difficulty swallowing, hand paresthesia, cough, and chest tightness. The next morning the patient left against medical advice but returned to the emergency department in the afternoon with worsening symptoms. The patient's wife reported that he had been bitten by a mongoose during the first week of October, but had not sought care for the bite. While being transferred to the intensive care unit, the patient went into cardiac arrest and died. On December 3, rabies was confirmed from specimens collected during autopsy. PRDH conducted an initial rapid risk assessment, and five family members were started on rabies postexposure prophylaxis (PEP).

Given potential additional exposures, PRDH and CDC undertook contact investigations among additional community and family members $(\mathrm{N}=32)$ and hospital personnel $(\mathrm{N}=39)$ to identify persons who required PEP. After the contact investigations, two additional family members and two hospital staff members received PEP. PRDH recommends that persons with a history of a mongoose bite should seek medical care and receive PEP. Health care providers should maintain a high index of clinical suspicion for rabies, including taking a history of animal exposure and adhering to recommended infection control practices when examining and treating anyone with suspected rabies or with acute, progressive encephalitis. Despite a high prevalence of rabies among mongoose populations in Puerto Rico, this is the first rabies-associated death directly related to a mongoose bite in Puerto Rico. In 2003, a case of human rabies occurred in a person infected with a mongoose variant of the virus after a dog bite (1). This case represents only the third documented rabies death in Puerto Rico during the past century. In Puerto Rico, public health outreach activities should continue to educate members of the community on mongoose-associated rabies and PEP.

\section{Case Report}

The patient, a man aged 54 years, was a resident of southeastern Puerto Rico. On the evening of November 30, he came to a local emergency department with fever, difficulty swallowing, hand paresthesia, cough, and chest tightness. He had refused most food and drink for the preceding 5 days and had difficulty managing his oral secretions. No history of animal exposure was elicited. He was given a preliminary diagnosis of lower respiratory tract infection and started empirically on antibiotics and antiemetics. A subsequent chest radiograph and computed tomography scan of his head were performed, which were both normal. The next morning, the patient left the hospital against medical advice.

The patient returned to the emergency department in the afternoon with worsening symptoms. The patient's wife, who accompanied him on this visit, reported that during the first week of October he had been bitten by a mongoose while tending to a chicken coop located on their property; he had not sought care after the encounter. Clinical suspicion of rabies triggered notification to $\mathrm{PRDH}$. Shortly thereafter, the patient experienced cardiac arrest while being transferred to the intensive care unit and could not be resuscitated.

An autopsy was performed on December 2 at the Puerto Rico Institute for Forensic Sciences, and specimens were submitted to the PRDH Public Health Laboratory for rabies testing. On December 3, results of direct fluorescent antibody testing were positive for rabies infection. Additional specimens sent to CDC for confirmatory testing were positive by direct fluorescent antibody and reverse transcription-polymerase chain reaction, and antigenic typing and sequence analysis were consistent with Caribbean mongoose rabies virus variant.

\section{Public Health Investigation}

Upon notification of the suspected case on December 1, PRDH collaborated with CDC's Poxvirus and Rabies Branch. An initial rapid risk assessment conducted by PRDH identified five family members who had potential exposures through close contact with the deceased patient, and all five family members were started on PEP. These persons included the patient's immediate family and household members.

Beginning on December 9, PRDH and CDC initiated contact investigations among additional family and community members as well as hospital personnel to determine other persons with potential exposure who required PEP. Based on the Advisory Committee on Immunization Practices guidelines for rabies virus exposures, PEP is recommended for persons with contact with the patient's saliva, tears, or cerebrospinal fluid to open wounds or mucous membranes during the infectious period ( 2 weeks before symptom onset) (2). A total of 76 contacts were 
evaluated for their risk for exposure, including two additional family members who required PEP because of exposure to the patient while he was hospitalized (Table). Among the 37 family and community contacts, median age was 33 (range $=1-78$ ), and 20 (54\%) were female. Municipality of residence was the same as that of the deceased patient for the 34 community members who reported place of residence. In total, seven (19\%) of the 37 family and community members received PEP.

Thirty-nine hospital personnel were reported to have had contact with the patient. These staff members worked in various positions, including intensive care, respiratory therapy, and patient transport. Median age was 35 years (range $=23-65$ ), and $18(46 \%)$ were female. Through the contact investigation, two $(5 \%)$ of the hospital staff members who had contact with the patient received PEP because they had exposures to the patient's saliva onto open wounds or mucous membranes. These exposures resulted from not wearing gloves or masks in the situations indicated in standard precautions (3), namely intubation and management of oral secretions.

After the contact investigations, education and outreach were conducted to inform community members and hospital personnel about rabies. PRDH designed and distributed educational materials to address the most frequently asked questions and held an informational session with the community to promote open discussion. In addition, hospital personnel participated in a debriefing that highlighted the need for appropriate use of standard precautions with all patients, regardless of suspected diagnosis.

\section{Discussion}

This is the first reported case of human rabies associated with a mongoose bite in North America. Mongooses were introduced from India to the Caribbean, including Puerto Rico, during the 19th century to control rat populations in sugarcane fields ( 4 ) and have become the principal reservoir of rabies in Puerto Rico, accounting for nearly $75 \%$ of all animal rabies cases $(5,6)$. In Puerto Rico, mongoose-associated rabies virus is phylogenetically linked to the North Central skunk and cosmopolitan dog variants (7). Seroprevalence of rabies virus-neutralizing antibodies in the mongoose population is estimated at $40 \%(8)$. Seventy-five mongoose bites were reported in 2014 (1.9 bites per 100,000 persons); during 2005-2008, 97\% of 151 submitted animal specimens after mongoose bites were positive for rabies virus. ${ }^{*} \mathrm{PRDH}$ recommends rabies PEP after all mongoose bites if the animal is not available for testing, and an estimated $95 \%$ of persons reporting mongoose bites receive PEP.

This case highlights the need for increased public awareness for the potential for mongoose-related rabies in Puerto Rico. The standardized risk assessment tool used in the contact investigations ensured that contacts with exposures promptly received PEP, thus mitigating costs from indiscriminate use of PEP. This tool could be adapted for use in other rabies exposure risk assessments in Puerto Rico or elsewhere. Health care providers should routinely assess for animal exposures in the medical history and maintain a high index of suspicion for rabies when animal exposure has occurred or is suspected. More generally, universal use and monitoring of standard precautions are necessary to minimize risk for exposures to infectious diseases in health care settings. Occupational exposures to rabies in health care settings frequently occur as a breach of standard precautions (9). Among hospital personnel interviewed for this investigation, only two (5\%) had an exposure, and both received PEP.

* Rivera-García B. Profile of mongoose inflicted bite injuries and PEP referral in Puerto Rico during fiscal years 2005-2008. Presented at Rabies in the Americas XX Conference, October 20, 2009, Québec, Canada.

TABLE. Characteristics of hospital and community contacts of human rabies case — Puerto Rico, 2015

\begin{tabular}{|c|c|c|c|c|c|c|}
\hline \multirow[b]{2}{*}{ Characteristic } & \multicolumn{3}{|c|}{ Hospital $(\mathrm{N}=39)$} & \multicolumn{3}{|c|}{ Community $(\mathrm{N}=37)$} \\
\hline & Unexposed & Exposed* & Total & Unexposed & Exposed* & Total \\
\hline Median age (range) (yrs) & $35(23-65)$ & $46(34-58)$ & $35(23-65)$ & $33(6-78)$ & $33(1-56)$ & $33(1-78)$ \\
\hline Male, no. (\%) & $20(51)$ & $1(3)$ & $21(54)$ & $13(76)$ & $4(24)$ & $17(46)$ \\
\hline \multicolumn{7}{|l|}{ Relationship to patient, no. (\%) } \\
\hline Physician & $8(22)$ & $1(50)$ & $9(23)$ & NA & NA & NA \\
\hline Nurse & $11(30)$ & $1(50)$ & $12(31)$ & NA & NA & NA \\
\hline Medical student & $5(14)$ & 0 & $5(13)$ & NA & NA & NA \\
\hline Respiratory therapist & $5(14)$ & 0 & $5(13)$ & NA & NA & NA \\
\hline Radiology technician/Patient escort & $4(11)$ & 0 & $4(10)$ & NA & NA & NA \\
\hline Janitorial staff & $4(11)$ & 0 & $4(10)$ & NA & NA & NA \\
\hline Immediate family & NA & NA & NA & $1(3)$ & $5(71)$ & $6(16)$ \\
\hline Relatives and friends & NA & NA & NA & $29(97)$ & $2(29)$ & $31(84)$ \\
\hline Total & $37(100)$ & $2(100)$ & 39 & $30(100)$ & $7(100)$ & 37 \\
\hline
\end{tabular}

Abbreviation: $\mathrm{NA}=$ not applicable.

* Based on Advisory Committee on Immunization Practices recommendations for postexposure prophylaxis. 


\section{Summary}

What is already known about this topic?

Human rabies associated with a mongoose encounter has never been reported in the United States or U.S. territories; however, studies by the U.S. Department of Agriculture indicate rabies seropositivity of approximately $40 \%$ among the Puerto Rican mongoose population. Because of the public health risk, Puerto Rico provides rabies postexposure prophylaxis (PEP) to any patient who experiences a mongoose bite.

What is added by this report?

A man aged 54 years who was bitten by a mongoose in October 2016 was the first person to acquire rabies from a mongoose in the United States or U.S. territories, confirming mongoose rabies as a public health threat. Limited awareness of rabies prevention and symptoms of the disease by the general public and health care personnel was likely a contributing factor in the exposures to the patient that required PEP.

What are the implications for public health practice?

This case highlights the importance of public and health care provider awareness of rabies to prevent adverse outcomes after exposures and reduce unnecessary exposures. This awareness includes maintaining a higher suspicion for zoonotic diseases by including animal exposures in the medical history. Universal use and monitoring of standard precautions in health care settings are necessary to minimize risk for occupational exposure to infectious diseases such as rabies when the nature of the illness is unknown.

Public health measures to reduce the risk for human rabies should include increased resources for primary prevention, including routine pet vaccination (canine rabies in Puerto Rico results from transmission from mongooses) and preexposure prophylaxis for persons at highest risk. Community education should highlight measures to avoid bites from pets and wildlife. Effective oral rabies vaccine baits targeting mongooses might also be considered as they become commercially available (10). Interventions should focus on areas with known human-mongoose contacts, as determined by overlaying bite surveillance data and population density. Secondary prevention measures should be aimed at increasing awareness of the need for medical evaluation and PEP after any mongoose bite.

\section{Acknowledgments}

Jesús Hernández Burgos, Puerto Rico Department of Health; Nelson Hernandez, Puerto Rico Institute for Forensic Sciences; San Lucas Hospital de Guayama, Guayama, Puerto Rico.

\footnotetext{
${ }^{1}$ Epidemic Intelligence Service, Division of Scientific Education and Professional Development, CDC; ${ }^{2}$ Poxvirus and Rabies Branch, National Center for Emerging and Zoonotic Infectious Diseases, CDC; ${ }^{3}$ Puerto Rico Department of Health; ${ }^{4}$ Office for State, Tribal, Local, and Territorial Support, CDC; ${ }^{5}$ Puerto Rico Institute for Forensic Sciences; ${ }^{6}$ Division of State and Local Readiness, Office of Public Health Preparedness and Response, CDC.
}

Corresponding author: Ashley Styczynski, astyczynski@cdc.gov, 404-661-6663.

\section{References}

1. Petersen BW, Rupprecht CE. Human rabies epidemiology and diagnosis [Chapter 10]. In: Tkachev S, ed. Non-flavivirus encephalitis. Rijeka, Crotia: InTech Open Science; 2011.

2. Manning SE, Rupprecht CE, Fishbein D, et al.; Advisory Committee on Immunization Practices Centers for Disease Control and Prevention (CDC). Human rabies prevention-United States, 2008: recommendations of the Advisory Committee on Immunization Practices. MMWR Recomm Rep 2008;57(No. RR-3).

3. Garner JS; The Hospital Infection Control Practices Advisory Committee. Guideline for isolation precautions in hospitals. Infect Control Hosp Epidemiol 1996;17:53-80. http://dx.doi.org/10.2307/30142367

4. Tierkel ES, Arbona G, Rivera A, de Juan A. Mongoose rabies in Puerto Rico. Public Health Rep 1952;67:274-8. http://dx.doi.org/10.2307/4588059

5. Dyer JL, Yager P, Orciari L, et al. Rabies surveillance in the United States during 2013. J Am Vet Med Assoc 2014;245:1111-23. http://dx.doi. org/10.2460/javma.245.10.1111

6. Krebs JW, Smith JS, Rupprecht CE, Childs JE. Rabies surveillance in the United States during 1998. J Am Vet Med Assoc 1999;215:1786-98.

7. Nadin-Davis SA, Velez J, Malaga C, Wandeler AI. A molecular epidemiological study of rabies in Puerto Rico. Virus Res 2008;131:8-15. http://dx.doi.org/10.1016/j.virusres.2007.08.002

8. Berentsen AR, Johnson SR, Gilbert AT, VerCauteren KC. Exposure to rabies in small Indian mongooses (Herpestes auropunctatus) from two regions in Puerto Rico. J Wildl Dis 2015;51:896-900. http://dx.doi. org/10.7589/2015-01-016

9. Kan VL, Joyce P, Benator D, et al. Risk assessment for healthcare workers after a sentinel case of rabies and review of the literature. Clin Infect Dis 2015;60:341-8. http://dx.doi.org/10.1093/cid/ciu850

10. Blanton JD, Meadows A, Murphy SM, et al. Vaccination of small Asian mongoose (Herpestes javanicus) against rabies. J Wildl Dis 2006;42:663-6. http://dx.doi.org/10.7589/0090-3558-42.3.663 